\title{
P,T-Violating Nuclear Matrix Elements in the One-Meson Exchange Approximation
}

\author{
I.S. Towner and A.C. Hayes \\ AECL Research, Chalk River Laboratories, Chalk River, Ontario, Canada K0J 1J0
}

(June 15, 2021)

\begin{abstract}
Expressions for the P,T-violating NN potentials are derived for $\pi, \rho$ and $\omega$ exchange. The nuclear matrix elements for $\rho$ and $\omega$ exchange are shown to be greatly suppressed, so that, under the assumption of comparable coupling constants, $\pi$ exchange would dominate by two orders of magnitude. The ratio of $\mathrm{P}, \mathrm{T}$-violating to $\mathrm{P}$-violating matrix elements is found to remain approximately constant across the nuclear mass table, thus establishing the proportionality between time-reversal-violation and parity-violation matrix elements. The calculated values of this ratio suggest a need to obtain an accuracy of order $5 \times 10^{-4}$ for the ratio of the PT-violating to P-violating asymmetries in neutron transmission experiments in order to improve on the present limits on the isovector pion coupling constant.
\end{abstract}

PACS: 11.30.Er,13.75.Cs,21.30.+y,24.80.-x 


\section{INTRODUCTION}

Simultaneous violation of parity conservation and time reversal invariance (P,T-violation) in the low-energy nucleon-nucleon interaction can be described, in analogy with the description of P-violation, in terms of nonrelativistic potentials derived from single-meson exchange diagrams involving the lightest pseudoscalar and vector mesons [1]. We consider here $\pi, \rho$ and $\omega$ mesons. The strength of the P,T-violation in the N-N interaction is then parameterised in terms of the coupling constants, $\bar{g}_{M N N}^{(I) \prime}$, characterising the $N \rightarrow N M$ matrix elements of the various isospin $(I)$ components. We consider the forms for the Lagrangian describing the $N \rightarrow N M$ vertex and find in a calculation in nuclei described by a closed-shell-plus-one configuration that the contribution to $\mathrm{P}, \mathrm{T}$-violation from all isospin components of $\rho$-exchange are identically zero for charge-symmetric $(N=Z)$ closed-shell cores. A similar result has been found [1,2] to hold true for the $I=0, I=2$ components of $\pi$-exchange. In heavy nu-

clei with closed-shell cores that are not charge symmetric, assuming equal couplings $\bar{g}_{M N N}^{(I) \prime}$, these terms together with the $\omega$-exchange terms remain small compared to the dominant term coming from the $I=1$ component of $\pi$-exchange.

$\mathrm{P}, \mathrm{T}$-violation may be studied in neutron transmission [3] and gamma-decay [4] [] experiments on oriented nuclei. In both cases P,T-violation is accompanied by P-violation alone. Consider a simple $\gamma$-decay example [1,5] where the initial nuclear state is of mixed parity due to the presence of P-violation and P,T-violation in the $\mathrm{NN}$-interaction, while the final state to a good approximation remains a state of definite parity. Let $\mid a>$ and $\mid b>$ denote the initial and final nuclear states in the absence of P- and P,T-violation. The potential, $V^{P}+V^{P, T}$, has the effect of changing $\mid a>$ to a state $\mid A>$ and $\mid b>$ to a state $\mid B>$, which under these assumptions are given by

$$
\begin{gathered}
|A>\simeq| a>+\sum_{a^{\prime}} \frac{\mid a^{\prime}>}{E_{a}-E_{a^{\prime}}} \times \\
\left(<a^{\prime}\left|V^{P}\right| a>+<a^{\prime}\left|V^{P, T}\right| a>\right) \\
|B>\simeq| b>
\end{gathered}
$$


where the state $\mid a^{\prime}>$ is of the same angular momentum as $\mid a>$ but of opposite parity. The $\mathrm{P}, \mathrm{T}$-violating effect is then proportional to

$$
A_{P, T} \simeq \operatorname{Im} \frac{<B\left\|\pi^{\prime} L^{\prime}\right\| A>}{<B\|\pi L\| A>},
$$

where $\pi^{\prime} L^{\prime}$ is the irregular multipole now available due to P,T-violation while $\pi L$ is the regular multipole present in the $|a>\rightarrow| b>+\gamma$ transition. A P-violating effect in the same transition is proportional to

$$
A_{P} \simeq R e \frac{<B\left\|\pi^{\prime} L^{\prime}\right\| A>}{<B\|\pi L\| A>} .
$$

It is useful to consider the ratio of these effects, which becomes proportional to the matrix elements of $V^{P}$ and $V^{P, T}$, viz [1]

$$
\frac{A_{P, T}}{A_{P}}=-i \frac{<a^{\prime}\left|V^{P, T}\right| a>}{<a^{\prime}\left|V^{P}\right| a>}+\cdots
$$

Here the ellipsis indicates that effective T-violation contributions induced by T-invariant higher-order processes have been omitted. It is important that these be estimated [6] before any comparison is made between theory and experiment, but they are not relevant for the discussion presented here. Herczeg [1] argues that if a calculation of $\left\langle a^{\prime}\left|V^{P, T}\right| a\right\rangle$ is not available, a rough estimate of the ratio $A_{P, T} / A_{P}$ combined with a measured value of the P-violating effect may give a better estimate of $<a^{\prime}\left|V^{P, T}\right| a>$ than a rough estimate of $<a^{\prime}\left|V^{P, T}\right| a>$ itself. To this end, Herczeg defines

$$
-i \frac{<a^{\prime}\left|V^{P, T}\right| a>}{<a^{\prime}\left|V^{P}\right| a>}=\kappa^{(1)} \frac{\bar{g}_{\pi N N}^{(1) \prime}}{g_{\rho N N}^{(0) \prime}} .
$$

Here it is assumed that P-violation occurs primarily through isoscalar $(I=0) \rho$-exchange characterized by the coupling constant $g_{\rho N N}^{(0) \prime}$. In the work of Desplanques, Donoghue and Holstein [7] (DDH), an isovector $(I=1) \pi$-exchange term is shown potentially to be of comparable importance in P-violation. However the limits on measurements [8] of circular polarisation of a selected $\gamma$-transition in ${ }^{18} F$, which is sensitive only to the isovector component of the P-violation, is found to be smaller than the DDH 'best estimate' and at present 
there is only an upper limit. Thus, in this analysis, we consider only isoscalar $\rho$-exchange contributing to P-violation.

Our goal here is to derive the potentials for the $\rho$ - and $\omega$ - exchange P,T-violating NN potentials and to determine the strength of their nuclear matrix elements relative to those for $\pi$ - exchange. We use these results to estimate a reasonable range for the value of $\kappa^{(1)}$. One of our main interests is in studying the dependence of the P,T-violating matrix elements, and hence $\kappa^{(1)}$, on nuclear mass. A somewhat similar undertaking has be made by Griffiths and Vogel [9] for the one-pion exchange potential. These authors follow Herczeg and express the effective one-body interaction in terms of an asymptotic potential obtained assuming $m_{\pi} \rightarrow$ $\infty$. The true one-body P,T-violating potential is then related to the asymptotic potential through a suppression factor $\mathrm{F}$, and $\mathrm{F}$ is shown to have a large dependence on nuclear mass. In the present work we do not use this analytic approximation as we find the assumption of large meson mass not to be good, particularly in the case of the pion; rather we perform exact numerical evaluations of the matrix elements. We find both the P,T- or P- violating matrix elements and consequently $\kappa^{(1)}$ to be approximately mass independent. Thus, we conclude that any strong mass dependence obtained using asymptotic approximations do not apply to the true potentials. In this regard there is little advantage in choosing examples in heavy nuclei over light as candidates for experimental study. Of course, any advantage from dynamical enhancements, such as the smallness of the energy denominator in eq. (1), remains valid.

\section{THE P,T-VIOLATING INTERACTION}

In a meson-exchange model, the P,T-violating nucleon-nucleon interaction is given by one weak $\mathrm{P}$,T-violating $N \rightarrow N M$ vertex and one strong $\mathrm{P}, \mathrm{T}$-conserving $N \rightarrow N M$ vertex with a meson exchanged between the two. For $\pi$-exchange the weak vertices are described by Lagrangians:

$$
\mathcal{L}_{\pi N N}^{(I=0)}=\bar{g}_{\pi N N}^{(0) \prime} \bar{N} N \tau^{a} \phi_{\pi}^{a}
$$




$$
\begin{gathered}
\mathcal{L}_{\pi N N}^{(I=1)}=\bar{g}_{\pi N N}^{(1) \prime} \bar{N} N \phi_{\pi}^{z} \\
\mathcal{L}_{\pi N N}^{(I=2)}=\bar{g}_{\pi N N}^{(2) \prime} \bar{N} N\left(3 \tau^{z} \phi_{\pi}^{z}-\tau^{a} \phi_{\pi}^{a}\right),
\end{gathered}
$$

where $\bar{g}_{\pi N N}^{(I) \prime}$ are coupling constants, $N$ nucleon fields and $\phi_{\pi}$ pion fields. The Roman superscripts are Cartesian isospin indices and a repeated index, $a$, is summed over, but not the index, $z$. The strong interaction vertex is

$$
\mathcal{L}_{\pi N N}=i g_{\pi N N} \bar{N} \gamma_{5} N \tau^{a} \phi_{\pi}^{a}
$$

with $g_{\pi N N}$ the strong pion-nucleon coupling constant, and $\tau$ the Pauli isospin matrix. Combining one strong and one weak vertex Lagrangian with the pion propagator, taking a nonrelativistic limit of the vertex functions, and making a Fourier transform to coordinate space leads to the following expressions for a P,T-violating potential

$$
\begin{gathered}
V_{P, T}^{(I=0)}=\bar{g}_{\pi N N}^{(0) \prime} g_{\pi N N} \frac{m_{\pi}^{2}}{8 \pi M} \boldsymbol{\sigma}_{-} \cdot \hat{\boldsymbol{r}} \boldsymbol{\tau}_{\mathbf{1}} \cdot \boldsymbol{\tau}_{\mathbf{2}} Y_{1}\left(x_{\pi}\right), \\
V_{P, T}^{(I=1)}=\bar{g}_{\pi N N}^{(1) \prime} g_{\pi N N} \frac{m_{\pi}^{2}}{16 \pi M}\left[\boldsymbol{\sigma}_{+} \cdot \hat{\boldsymbol{r}} \tau_{-}^{z}+\boldsymbol{\sigma}_{-} \cdot \hat{\boldsymbol{r}} \tau_{+}^{z}\right] Y_{1}\left(x_{\pi}\right), \\
V_{P, T}^{(I=2)}=\bar{g}_{\pi N N}^{(1) \prime} g_{\pi N N} \frac{m_{\pi}^{2}}{8 \pi M} \boldsymbol{\sigma}_{-} \cdot \hat{\boldsymbol{r}}\left[3 \tau_{1}^{z} \tau_{2}^{z}-\boldsymbol{\tau}_{\mathbf{1}} \cdot \boldsymbol{\tau}_{\mathbf{2}}\right] Y_{1}\left(x_{\pi}\right),
\end{gathered}
$$

where $\boldsymbol{\sigma}_{+}=\boldsymbol{\sigma}_{1}+\boldsymbol{\sigma}_{2}, \boldsymbol{\sigma}_{-}=\boldsymbol{\sigma}_{1}-\boldsymbol{\sigma}_{2}$ and similarly for $\tau_{+}$and $\tau_{-}, m_{\pi}$ is the pion mass, and $M$ the nucleon mass. Here $\boldsymbol{r}=\boldsymbol{r}_{\mathbf{1}}-\boldsymbol{r}_{\mathbf{2}}, x_{\pi}=m_{\pi} r$ and $Y_{1}(x)=(1+1 / x) Y_{0}(x)$ with $Y_{0}(x)=e^{-x} / x$. This result has been given before [1,2].

Likewise for the vector mesons, the P,T-violating Lagrangians are

$$
\begin{gathered}
\mathcal{L}_{\rho N N}^{(I=0)}=i \bar{g}_{\rho N N}^{(0) \prime} \frac{1}{2 M} \bar{N} \sigma_{\mu \nu} \partial_{\nu} \gamma_{5} N \tau^{a} \rho_{\mu}^{a}, \\
\mathcal{L}_{\rho N N}^{(I=1)}=i \bar{g}_{\rho N N}^{(1) \prime} \frac{1}{2 M} \bar{N} \sigma_{\mu \nu} \partial_{\nu} \gamma_{5} N \rho_{\mu}^{z}, \\
\mathcal{L}_{\rho N N}^{(I=2)}=i \bar{g}_{\rho N N}^{(2) \prime} \frac{1}{2 M} \bar{N} \sigma_{\mu \nu} \partial_{\nu} \gamma_{5} N\left(3 \tau^{z} \rho_{\mu}^{z}-\tau^{a} \rho_{\mu}^{a}\right),
\end{gathered}
$$

where $\rho_{\mu}^{a}$ are rho-meson fields and $\sigma_{\mu \nu}=\left(\gamma_{\mu} \gamma_{\nu}-\gamma_{\nu} \gamma_{\mu}\right) / 2 i$. We are using the Pauli metric for Dirac matrices as discussed in DeWit and Smith 10. Note that the Lorentz form of 
these Lagrangians is that of an axial-vector that is odd under charge conjugation. They correspond to second-class axial currents [11] in the terminology of nuclear $\beta$-decay. The strong interaction vertex

$$
\mathcal{L}_{\rho N N}=i g_{\rho N N} \bar{N}\left(\gamma_{\mu}+i \frac{K_{V}}{2 M} \sigma_{\mu \nu} \partial_{\nu}\right) N \tau^{a} \rho_{\mu}^{a}
$$

where $K_{V}$ is the ratio of tensor to vector coupling constants. The corresponding P,Tviolating potentials in co-ordinate space are

$$
\begin{gathered}
V_{P, T}^{(I=0)}=-\bar{g}_{\rho N N}^{(0) \prime} g_{\rho N N} \frac{m_{\rho}^{2}}{8 \pi M} \boldsymbol{\sigma}_{-} \cdot \hat{\boldsymbol{r}} \boldsymbol{\tau}_{\mathbf{1}} \cdot \boldsymbol{\tau}_{\mathbf{2}} Y_{1}\left(x_{\rho}\right), \\
V_{P, T}^{(I=1)}=\bar{g}_{\rho N N}^{(1) \prime} g_{\rho N N} \frac{m_{\rho}^{2}}{16 \pi M}\left[\boldsymbol{\sigma}_{+} \cdot \hat{\boldsymbol{r}} \tau_{-}^{z}-\boldsymbol{\sigma}_{-} \cdot \hat{\boldsymbol{r}} \tau_{+}^{z}\right] Y_{1}\left(x_{\rho}\right), \\
V_{P, T}^{(I=2)}=-\bar{g}_{\rho N N}^{(2) \prime} g_{\rho N N} \frac{m_{\rho}^{2}}{8 \pi M} \boldsymbol{\sigma} \boldsymbol{\sigma}_{-} \cdot \hat{\boldsymbol{r}}\left(3 \tau_{1}^{z} \tau_{2}^{z}-\boldsymbol{\tau}_{\mathbf{1}} \cdot \boldsymbol{\tau}_{\mathbf{2}}\right) Y_{1}\left(x_{\rho}\right) .
\end{gathered}
$$

The overall form of these potentials is the same as for $\pi$-exchange except for one key difference in the isovector potential. There the relative sign between the two terms is minus for $\rho$ exchange and plus for $\pi$-exchange. Lastly, for $\omega$-exchange we make substitutions $\tau^{a} \rho_{\mu}^{a} \rightarrow \omega_{\mu}$ for isoscalar and $\rho_{\mu}^{z} \rightarrow \tau^{z} \omega_{\mu}$ for isovector Lagrangians and obtain the following P,T-violating potentials

$$
\begin{gathered}
V_{P, T}^{(I=0)}=-\bar{g}_{\omega N N}^{(0) \prime} g_{\omega N N} \frac{m_{\omega}^{2}}{8 \pi M} \boldsymbol{\sigma}_{-} \cdot \hat{\boldsymbol{r}} Y_{1}\left(x_{\omega}\right) \\
V_{P, T}^{(I=1)}=-\bar{g}_{\omega N N}^{(1) \prime} g_{\omega N N} \frac{m_{\omega}^{2}}{16 \pi M}\left[\boldsymbol{\sigma}_{+} \cdot \hat{\boldsymbol{r}} \tau_{-}^{z}+\boldsymbol{\sigma}_{-} \cdot \hat{\boldsymbol{r}} \tau_{+}^{z}\right] Y_{1}\left(x_{\omega}\right)
\end{gathered}
$$

Note in this case there is a relative plus sign between the two isovector terms. There is no isotensor potential for $\omega$-exchange.

To estimate which components of the P,T-violating interaction are important, we compute matrix elements of $V_{P, T}$ for closed-shell-plus-one configurations. This is equivalent to determining effective one-body potentials. The computation boils down to an evaluation of two-body matrix elements between the valence nucleon and one of the nucleons in the closed-shell core summed over all the nucleons in the core. Let $a$ and $a^{\prime}$ be two quantum 
states of the valence nucleon (of the same angular momentum, $j_{a}$, but opposite parity), and $c$ the quantum states of the occupied orbits in the core, then for charge-symmetric cores

$$
\begin{aligned}
& <a^{\prime} m_{a}\left|V_{P, T}^{(I)}\right| a m_{a}>=\sum_{\substack{c \\
J_{1} J_{2} T_{1} T_{2}}} \frac{\hat{J}_{1} \hat{J}_{2} \hat{T}_{1} \hat{T}_{2}}{\left(4 j_{a}+2\right)} U\left(\frac{1}{2} \frac{1}{2} I T_{2} ; T_{1} \frac{1}{2}\right) \\
& <\frac{1}{2} m_{a} I 0 \mid \frac{1}{2} m_{a}><\left(a^{\prime} c\right) J_{1} T_{1}\left\|V_{P, T}^{(I)}\right\|(a c) J_{2} T_{2}>
\end{aligned}
$$

where $\hat{J}=(2 J+1)^{1 / 2}$ and the $U$-coefficient is a recoupling coefficient of three angular momenta. Our notation is that of Brink and Satchler [12] with the matrix elements reduced in both spin and isospin spaces. The two-body matrix element on the right-hand side of eq. (14) is antisymmetrised; such that the sum over core states includes both the direct and exchange terms. From selection rules, the U-coefficient is zero when $I=2$, thus there is no contribution to the matrix element from isotensor components of the interaction for charge-symmetric cores. Furthermore matrix elements of $\boldsymbol{\sigma}_{-} \boldsymbol{\tau}_{\mathbf{1}} \cdot \boldsymbol{\tau}_{\mathbf{2}}$ are zero, and matrix elements of $\boldsymbol{\sigma}_{-} \tau_{+}^{z}$ and $\boldsymbol{\sigma}_{+} \tau_{-}^{z}$ are equal when summing over charge-symmetric cores. Thus the $\rho$-exchange interaction gives no contribution in this case. As noted in [1,2] a similar result holds for the $\mathrm{I}=0,2$ components of the $\pi$-exchange interaction.

For a charge non-symmetric core $(N \neq Z)$, eq. (14) is generalised to read

$$
\begin{gathered}
<a^{\prime} m_{a}\left|V_{P, T}^{(I)}\right| a m_{a}>=\sum_{\substack{c \\
J_{1} T_{2} T_{1} T_{2} \\
m_{c} M_{1} M_{2}}} \frac{\hat{J}_{1} \hat{J}_{2}}{\left(2 j_{a}+1\right)} \times \\
<\frac{1}{2} m_{a} \frac{1}{2} m_{c}\left|T_{1} M_{1}><\frac{1}{2} m_{a} \frac{1}{2} m_{c}\right| T_{2} M_{2}> \\
<T_{2} M_{2} I 0 \mid T_{1} M_{1}><\left(a^{\prime} c\right) J_{1} T_{1}\left\|V_{P, T}^{(I)}\right\|(a c) J_{2} T_{2}>,
\end{gathered}
$$

where Clebsch-Gordan coefficients replace the U-coefficient. Here $m_{a}$ is the $z$-component of the valence nucleon's isospin quantum number and is $-1 / 2$ for a neutron and $+1 / 2$ for a proton. Now terms that gave zero contribution for charge-symmetric cores give a finite but generally small contribution in heavy nuclei. Some sample calculations are given in Table — for $j_{a}=1 / 2, p$ - and $s$ - states. 
Our calculations are made with harmonic oscillator wavefunctions. However there is one drawback to this choice; the eigenfunctions of the harmonic oscillator potential are not the eigenfunctions of the nucleon-nucleon interaction. This is particularly important in the relative coordinate, $r$, where the nucleon-nucleon interaction is known to have a strong short-range repulsion, which makes the relative wavefunction go rapidly to zero as $r \rightarrow 0$, more rapidly than given by uncorrelated oscillator functions. Thus to incorporate this piece of many-body physics in a simple way it is quite common to modify two-body operators by multiplying them by a short-range correlation function. Thus we write

$$
\hat{V}_{P, T}^{(I)}(r)=V_{P, T}^{(I)}(r) \hat{g}(r)
$$

where $\hat{g}(r)$ is some function that tends to zero as $r \rightarrow 0$ and tends to unity for large $r$. The precise choice of $\hat{g}(r)$ becomes part of the model dependence at short distances. This choice is not critical if the operator is of long-range, such as the pion-exchange interactions, but is much more critical for the shorter-ranged, heavy-meson exchange interactions. Our results in Table 1 adopt the choice made by Adelberger and Haxton [13], who in their work on parity violation in nuclei used $\hat{g}(r)=\left[1-\exp \left(-a r^{2}\right)\left(1-b r^{2}\right)\right]^{2}$ with $a=1.1 \mathrm{fm}^{-2}$ and $b=0.68 f m^{-2}$

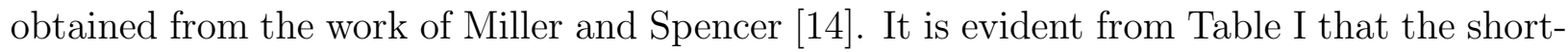
range correlation function significantly reduces the contribution from heavy mesons. Thus, providing all the unknown coupling constants $\bar{g}_{M N N}^{(I) \prime}$ are of comparable magnitude, it can be asserted that the isovector pion-exchange component will dominate P,T-violation in nuclei.

An estimate of the coupling constants has recently been provided by Gudkov, He and MacKellar [15]. These authors point out that, in general, a first principles calculation of P,T-violation would be very difficult. However, beginning with an effective low-energy P,Tviolating Lagrangian at the quark level for particular models of CP violation, they have estimated the P,T-violating coupling constants, $\bar{g}_{M N N}^{(I) \prime}$, of the nucleon-nucleon interaction using a factorization approximation and the vector dominance hypothesis. Their main conclusion is that for all types of models of $\mathrm{CP}$ violation in the one-meson-exchange approximation the contributions to the P,T-violating nucleon-nucleon interaction from pseudoscalar mesons are 
larger than the contributions from vector mesons by about one order of magnitude.

\section{THE RATIO $\kappa^{(1)}$}

We now return to the evaluation of the ratio of $\mathrm{P}, \mathrm{T}$-violation to $\mathrm{P}$-violation matrix elements of eq. (6). We assume that P-violation principally comes from the isoscalar $\rho$ exchange potential [7, 13]

$$
V_{\rho}=-i g_{\rho N N}^{(0) \prime}\left(1+K_{V}\right) \frac{m_{\rho}^{2}}{4 \pi M} i \boldsymbol{\sigma}_{\mathbf{1}} \times \boldsymbol{\sigma}_{\mathbf{2}} \cdot \hat{\boldsymbol{r}} \boldsymbol{\tau}_{\mathbf{1}} \cdot \boldsymbol{\tau}_{\mathbf{2}} Y_{1}\left(x_{\rho}\right),
$$

where we have neglected a smaller non-local term. The weak coupling constant $g_{\rho N N}^{(0) \prime}$ is the same as $h_{\rho}^{0}$ in the notation of DDH [7]. The ratio therefore becomes

$$
\begin{aligned}
\kappa^{(1)}= & \frac{1}{4} \frac{g_{\pi N N}}{g_{\rho N N}\left(1+K_{V}\right)} \frac{m_{\pi}^{2}}{m_{\rho}^{2}} \times \\
& \frac{\left\langle\left(\boldsymbol{\sigma}_{+} \cdot \hat{\boldsymbol{r}} \boldsymbol{\tau}_{-}+\boldsymbol{\sigma}_{-} \cdot \hat{\boldsymbol{r}} \boldsymbol{\tau}_{+}\right) Y_{1}\left(x_{\pi}\right)\right\rangle}{\left\langle i \boldsymbol{\sigma}_{\mathbf{1}} \times \boldsymbol{\sigma}_{\mathbf{2}} \cdot \hat{\boldsymbol{r}} \boldsymbol{\tau}_{\mathbf{1}} \cdot \boldsymbol{\tau}_{\mathbf{2}} Y_{1}\left(x_{\rho}\right)\right\rangle} \frac{\bar{\omega}_{\pi}}{\omega_{\rho}}
\end{aligned}
$$

where $\bar{\omega}_{\pi}, \omega_{\rho}$ are reduction factors accounting for short-range correlations.

Our exact numerical results for the ratio of the P,T-violating to P-violating matrix elements, (incorporating the reduction factors $\bar{\omega}_{\pi}$ and $\omega_{\rho}$ explicitly as decribed in eq. (16)), are summarized in Table 【. There we give some sample neutron matrix elements in closed-shellplus-one configurations for $j_{a}=1 / 2, p$ - and $s$-states. Significantly, the $\kappa^{(1)}$ values display very little mass dependence, and range from 2 to 5 .

To understand our results summarized in Tables I and II let us consider the simple case a neutron in a closed-shell-plus-one configuration for charge symmetric cores, eq. (14). Then, for the $\pi$-exchange term, the exchange component of the two-body matrix elements is identically zero, while for the $\rho$-exchange term the direct component is zero. The spin and isospin sums are trivially evaluated: $\left.<\boldsymbol{\sigma}_{+} \tau_{-}\right\rangle=$ $\left.<\boldsymbol{\sigma}_{-} \tau_{+}\right\rangle=12$ and $\left\langle i \boldsymbol{\sigma}_{\mathbf{1}} \times \boldsymbol{\sigma}_{\mathbf{2}} \boldsymbol{\tau}_{\mathbf{1}} \cdot \boldsymbol{\tau}_{\mathbf{2}}\right\rangle=-18$ and the angle integral $\langle\hat{\boldsymbol{r}}>$ cancels in the ratio to give 


$$
\kappa^{(1)}=-\frac{1}{3} \frac{g_{\pi N N}}{g_{\rho N N}\left(1+K_{V}\right)} \frac{m_{\pi}^{2}}{m_{\rho}^{2}} \frac{<Y_{1}\left(x_{\pi}\right)>\bar{\omega}_{\pi}}{<Y_{1}\left(x_{\rho}\right)>} .
$$

It remains to evaluate the radial integrals, $\left\langle Y_{1}\left(x_{\pi}\right)\right\rangle$. Consider the integral evaluated between $0 s$ and $0 p$ oscillator functions of length parameter, $b$. The exact value of the integral is

$$
\begin{gathered}
<Y_{1}(x)>=\left(\frac{2}{3}\right)^{1 / 2}\left[z e^{z^{2}} \operatorname{erfc}(z)+\frac{1}{\pi^{1 / 2}}\left(\frac{1}{2 z^{2}}-1\right)\right] \\
\Rightarrow\left(\frac{2}{3}\right)^{1 / 2} \frac{3}{4 \pi^{1 / 2}} \frac{1}{z^{4}} \quad \text { for } \quad z>>1,
\end{gathered}
$$

where $z=m b / \sqrt{2}$ and $m$ the meson mass. Thus, in the limit of large meson mass the radial integrals, $<Y_{1}\left(x_{\pi}\right)>$ scales as $1 / m^{4}$. The short-range correlation function cuts down matrix elements of $\rho$-meson range by roughly a factor of three more than those of $\pi$-meson range, $\bar{\omega}_{\pi} / \omega_{\rho} \simeq 3$

The approximate scaling of the radial integrals with $1 / m^{4}$ is largely responsible for the large difference in the magnitudes of the $\pi$ - and vector meson P,T-violating matrix elements. We note, however, that for pion range, $m_{\pi}=0.7 \mathrm{fm}^{-1}$, the inequality $z>>1$ is not satisfied, not even in heavy nuclei since $b$ only varies gently with nuclear mass as $A^{1 / 6}$. This results in the scaling approximation, when taken at face value, leading to an overestimate in the ratio $\kappa^{(1)}$ by a factor of three. More detailed calculations reduce $\kappa^{(1)}$ even further, to give the values listed in the Table II.

In addition to examining [9] the effective one-body P,T-violating potential, Griffiths and Vogel [5] has also calculated $\kappa^{(1)}$ for specific rare-earth nuclei, using the quasiparticle random phase approximation. They find $\kappa^{(1)}$ values in the range 1 to 6 , which is similar to the present range of 2 to 5 . Since $\kappa^{(1)}$ seems to be determined to within quite a small range independent of nuclear structure considerations, then a degree of proportionality between the matrix elements of the P,T- and P-violating potentials seems to be established. 


\section{P,T-VIOLATION IN NEUTRON TRANSMISSION}

There is much interest in the possibility of probing $\mathrm{P}, \mathrm{T}$-violation in the transmission of polarized neutrons through polarized nuclear targets [3]. Neutron transmission measurements on unpolarized targets have proved to be powerful probes of P-violation. There the scattering cross sections of low-energy neutrons from nuclei at $p$-wave resonances exhibit very large parity violating longitudinal asymmetries. The P-violating asymmetries, which are defined as the fractional difference of the resonance cross section for neutrons polarized parallel and antiparallel to their momentum, $P_{P}=\sigma^{+}-\sigma^{-} / \sigma^{+}+\sigma^{-}$, arise from $s_{1 / 2}$ admixtures in the $p_{1 / 2}$ resonances, and can be as large as $10 \%$. In the case of polarized targets the asymmetry $P_{P, T}=\sigma^{\uparrow}-\sigma^{\downarrow} / \sigma^{\uparrow}+\sigma^{\downarrow}$, implies simultaneous P- and T-violation through a term in the scattering amplitude proportional to $\left\langle\boldsymbol{\sigma}>\cdot(\boldsymbol{k} \times<\boldsymbol{J}>)\right.$. Here $\sigma^{\uparrow}$ and

$\sigma^{\downarrow}$ are the total scattering cross-sections for neutrons polarized parallel and antiparallel to $(\boldsymbol{k} \times<\boldsymbol{J}>)$, and $\boldsymbol{\sigma}$ and $\boldsymbol{k}$ are the neutron spin and momentum and $\boldsymbol{J}$ is the target spin.

In analogy with eq. (5), the ratio of the P,T-violating to P-violating asymmetries in the neutron measurements can be related to the ratio of the matrix elements of the corresponding weak NN potentials. In a two-state mixing approximation the ratio is given by,

$$
\lambda=\frac{P_{P, T}}{P_{P}} \simeq-i \frac{<\psi_{s}\left|V^{P, T}\right| \psi_{p}>}{<\psi_{s}\left|V^{P}\right| \psi_{p}>},
$$

where $\psi_{s}$ and $\psi_{p}$ are compound nuclear $s$ - and $p$-wave resonance wave functions. To first order the ratio of the weak matrix elements between compound resonances can be approximated by the ratio of single-particle matrix elements, so that [1]],

$$
\lambda^{(I)} \approx \kappa^{(I)} \frac{\bar{g}_{\pi N N}^{(I) \prime}}{g_{\rho N N}^{(0) \prime}} .
$$

Herczeg has reviewed the experimental searches of electric dipole moments in atoms, molecules and the neutron, and barring cancellations between the different isospin amplitudes, deduces the following upper limits for the P,T-violating $\pi \mathrm{NN}$ constants

$$
\left|\bar{g}_{\pi N N}^{(0) \prime}\right| \lesssim 1.4 \times 10^{-11}
$$




$$
\begin{gathered}
\left|\bar{g}_{\pi N N}^{(1) \prime}\right| \lesssim 10^{-10}, \\
\left|\bar{g}_{\pi N N}^{(2) \prime}\right| \lesssim 1.4 \times 10^{-11} .
\end{gathered}
$$

To get a rough estimate of the accuracy needed in the neutron transmission experiments to improve on these limits we consider ${ }^{139} \mathrm{La}$, where a very large P-violating asymmetry $\left(P_{P} \sim 10^{-1}\right)$ has been observed. Combining Herczeg's limits with the values of $\kappa^{(0)}, \kappa^{(1)}$, and $\kappa^{(2)}$ from Tables I and II for $\mathrm{A}=138$, and taking $g_{\rho N N}^{(0) \prime}=-11.4 \times 10^{-7}$ gives,

$$
\begin{aligned}
& \left|\lambda^{(0)}\right| \lesssim 1.6 \times 10^{-5}, \\
& \left|\lambda^{(1)}\right| \lesssim 5 \times 10^{-4}, \\
& \left|\lambda^{(2)}\right| \lesssim 2.5 \times 10^{-5} .
\end{aligned}
$$

The enhanced sensitivity to the isovector coupling arises because the $I=1 \mathrm{P}, \mathrm{T}$-violating interaction is probed by all A nucleons, whereas the isoscalar and isotensor interactions are only probed by the excess neutrons [四]. This situation is the reverse of that in the neutron electric dipole experiments, where sensitivity to the isovector term is suppressed. A $P_{P, T} / \mathrm{P}_{P}$ $\sim 10^{-4}$ would then provide much tighter constraints on the isovector P,T-odd $\pi \mathrm{NN}$ coupling constant.

\section{CONCLUSIONS}

We have derived the PT-violating NN potentials for $\rho$ - and $\omega$-exchange and demonstrated quantitatively what has only been speculated qualitatively before, namely that $\rho$ and $\omega$ exchange give a negligible contribution to time-reversal violation compared to $\pi$ exchange, assuming comparable coupling constants. Under this assumption the heavy-meson matrix elements are about two orders of magnitude smaller than the $I=1 \pi$-exchange matrix elements. This is in sharp contrast to parity violation, where heavy-meson exchange is comparable to pion exchange. 
The supression of the vector-meson contributions to P,T-violation arises from a combination of factors. The first of these applies to $\rho$-exchange where the form of the P,T-violating potentials are the same as for $\pi$-exchange except for a crucial sign difference between the two terms contributing to the $I=1$ potential. For $N=Z$ cores these two terms are equal in magnitude. They add constructively for $\pi$-exchange but exactly cancel for $\rho$-exchange. Moving to heavier nuclei, where $N \neq Z$, breaks this cancellation to a small though not significant degree. The $I=0$ and $I=2 \rho$-exchange potentials have identical forms to the corresponding $\pi$-exchange potentials. Thus, as in the case of the $I=0$ and $I=2$ pion terms, they give zero contribution to P,T-violation in $N=Z$ systems, and contribute through the excess neutrons in heavy nuclei. We note, as can be seen from Table I, that the $I=0$ and $I=1 \omega$-exchange terms are not zero for charge-symmetric systems, owing to the different nature of the $\omega$-exchange potentials, eqs. (13).

The second source of suppression applies equally to $\rho$ - and $\omega$-exchange and is due to the approximate scaling of the radial matrix of $Y_{1}(x)$ with $1 / m^{4}$, so that the matrix elements of $V_{P, T}$ scale approximately as $1 / m^{2}$. This reduces the $\rho$ - and $\omega$-exchange matrix elements by about a factor of 25 relative to those for the pion. Finally, the short-range correlations function reduces the radial matrix elements for the heavy-mesons by an additional factor of about three.

$\mathrm{P}, \mathrm{T}$-violation in both $\gamma$-decay and neutron transmission experiments is always accompanied by P-violation alone, and the sensitivity of these searches is determined by the ratio of the P,T-violating to P-violating matrix elements. We have calculated this ratio, $\kappa^{(1)}$, for closed-shell-plus-one configurations and find it to be approximately independent of the nucleus under study. We find the calculated values of $\kappa^{(1)}$ to lie in the range 2 to 5 . This range is similar to the range 1 to 6 found by Griffiths and Vogel [5] in calculations for rare earth nuclei using the quasiparticle random phase approximation. This suggests that $\kappa^{(1)}$ is determined within quite a small range independent of detailed nuclear structure considerations. Thus, the degree of proportionality between time-reversal-violation and parity-violation matrix elements seems to have been established. 
The authors wish to thank Peter Herczeg for correspondence on the weak $\rho$-meson Lagrangians, and for valuable suggestions concerning the manuscript. 


\section{REFERENCES}

[1] P. Herczeg, Hyperfine Interact. 75, 127 (1992); P. Herczeg, in Tests of Time Reversal Invariance, eds. N.R. Roberson, C.R. Gould and J.D. Bowman (World Scientific, Singapore, 1987), pg. 24

[2] W.C. Haxton and E.M. Henley, Phys. Rev. Lett. 51, 1937 (1983)

[3] J.D. Bowman et al., in Fundamental Symmetries in Nuclei and Particles, ed. H. Henrikson and P. Vogel (World Scientific, Singapore, 1990), pg. 1

[4] F. Boehm, in Hyperfine Structure and Nuclear Reactions, ed. E. Matthias and D.A. Shirley (North-Holland, Amsterdam, 1968), pg. 279

[5] A. Griffiths and P. Vogel, Phys. Rev. C44, 1195 (1991)

[6] A. Griffiths and P. Vogel, Phys. Rev. C44, 1215 (1991)

[7] B. Desplanques, J.F. Donoghue and B.R. Holstein, Ann. Phys. (NY) 124, 449 (1980)

[8] H.C. Evans et al., Phys. Rev. Lett. 55, 791 (1985); M. Bini et al., Phys. Rev. Lett. 55, $795(1985)$

[9] A. Griffiths and P. Vogel, Phys. Rev. C43, 2844 (1991)

[10] B. deWit and J. Smith, Field Theory in Particle Physics (North-Holland, Amsterdam, 1986)

[11] T.D. Lee, Particle Physics and Introduction to Field Theory (Harwood Academic Publishers, New York, 1981); S. Weinberg, Phys. Rev. 112, 1375 (1958)

[12] D.M. Brink and G.R. Satchler, Angular Momentum (Clarendon, Oxford, 1968)

[13] E.G. Adelberger and W.C. Haxton, Annu. Rev. Nucl. Part. Sci. 35, 501 (1985)

[14] G.A. Miller and J.E. Spencer, Ann. Phys. (NY) 100, 562 (1976)

[15] V.P. Gudkov, X-G He, and B.H.J. McKellar, Phys. Rev. C47, 2365 (1993) 


\section{TABLES}

TABLE I. Contribution from each component of the two-body $V_{P, T}$ interaction to the $\mathrm{P}, \mathrm{T}$-violating matrix element in closed-shell-plus-one configuration in units of $\mathrm{eV} \times 10^{-4}$ for six choices of the closed-shell core. Matrix elements were calculated with harmonic oscillator wave functions with $\hbar \omega=45 A^{-1 / 3}-25 A^{-2 / 3} \mathrm{MeV}$. The Miller-Spencer [14] short-range correlation function was used. The weak interaction coupling constants, $\bar{g}_{M N N}^{(I) \prime}$, were set at $1 \times 10^{-11}$.

\begin{tabular}{|c|c|c|c|c|c|c|}
\hline & ${ }^{16} \mathrm{O}$ & ${ }^{40} \mathrm{Ca}$ & ${ }^{90} \mathrm{Zr}$ & ${ }^{138} \mathrm{Ba}$ & ${ }^{208} \mathrm{~Pb}$ & ${ }^{232} \mathrm{Th}$ \\
\hline & $\mathrm{N}=8$ & $\mathrm{~N}=20$ & $\mathrm{~N}=50$ & $\mathrm{~N}=82$ & $\mathrm{~N}=126$ & $\mathrm{~N}=142$ \\
\hline & $\mathrm{Z}=8$ & $\mathrm{Z}=20$ & $\mathrm{Z}=40$ & $\mathrm{Z}=56$ & $\mathrm{Z}=82$ & $\mathrm{Z}=90$ \\
\hline & $\underline{0 p-0 s}$ & $\underline{1 p-1 s}$ & $\underline{2 p-2 s}$ & $\underline{2 p-2 s}$ & $\underline{3 p-3 s}$ & $\underline{3 p-3 s}$ \\
\hline$\pi(\mathrm{I}=0)$ & - & - & -0.059 & -0.181 & -0.126 & -0.162 \\
\hline$\pi(\mathrm{I}=1)$ & 1.084 & 0.875 & 0.708 & 0.779 & 0.608 & 0.633 \\
\hline$\pi(\mathrm{I}=2)$ & - & - & 0.000 & -0.275 & -0.190 & -0.179 \\
\hline$\rho(\mathrm{I}=0)$ & - & - & 0.000 & 0.002 & 0.001 & 0.001 \\
\hline$\rho(\mathrm{I}=1)$ & - & - & -0.000 & -0.001 & -0.000 & -0.001 \\
\hline$\rho(\mathrm{I}=2)$ & - & - & 0.000 & 0.001 & 0.000 & 0.000 \\
\hline$\omega(\mathrm{I}=0)$ & 0.020 & 0.012 & 0.007 & 0.008 & 0.006 & 0.006 \\
\hline$\omega(\mathrm{I}=1)$ & -0.020 & -0.012 & -0.007 & -0.008 & -0.006 & -0.006 \\
\hline & $0 p-1 s$ & $1 p-2 s$ & $2 p-3 s$ & $2 p-3 s$ & $3 p-4 s$ & $3 p-4 s$ \\
\hline$\pi(\mathrm{I}=0)$ & - & - & 0.054 & 0.122 & 0.097 & 0.125 \\
\hline$\pi(\mathrm{I}=1)$ & -0.400 & -0.378 & -0.388 & -0.465 & -0.376 & -0.409 \\
\hline$\pi(\mathrm{I}=2)$ & - & - & 0.000 & 0.181 & 0.146 & 0.138 \\
\hline$\rho(\mathrm{I}=0)$ & - & - & -0.000 & -0.001 & -0.001 & -0.001 \\
\hline$\rho(\mathrm{I}=1)$ & - & - & 0.000 & 0.000 & 0.000 & 0.001 \\
\hline$\rho(\mathrm{I}=2)$ & - & - & 0.000 & -0.000 & -0.001 & -0.001 \\
\hline$\omega(\mathrm{I}=0)$ & -0.008 & -0.003 & -0.004 & -0.005 & -0.003 & -0.004 \\
\hline$\omega(\mathrm{I}=1)$ & 0.008 & 0.003 & 0.004 & 0.005 & 0.003 & 0.004 \\
\hline
\end{tabular}


TABLE II. Isovector $\pi$-exchange, $V_{P, T}$, and isoscalar $\rho$-exchange, $V_{P}$, matrix elements evaluated for a closed-shell-plus-one configuration for six choices of the closed-shell core. The weak interaction coupling constants are $\bar{g}_{\pi N N}^{(1) \prime}=1.0 \times 10^{-11}$ and $g_{\rho N N}^{(0) \prime}=-11.4 \times 10^{-7}$. Matrix elements were calculated with harmonic oscillator wavefunctions with $\hbar \omega=45 A^{-1 / 3}-25 A^{-2 / 3} \mathrm{MeV}$. The Miller-Spencer [14] short-range correlation function was used. The ratio, $\kappa^{(1)}$, is defined in eq. (6).

\begin{tabular}{|c|c|c|c|c|c|c|}
\hline & ${ }^{16} \mathrm{O}$ & ${ }^{40} \mathrm{Ca}$ & ${ }^{90} \mathrm{Zr}$ & ${ }^{138} \mathrm{Ba}$ & ${ }^{208} \mathrm{~Pb}$ & ${ }^{232} \mathrm{Th}$ \\
\hline & $\mathrm{N}=8$ & $\mathrm{~N}=20$ & $\mathrm{~N}=50$ & $\mathrm{~N}=82$ & $\mathrm{~N}=126$ & $\mathrm{~N}=142$ \\
\hline & $\mathrm{Z}=8$ & $\mathrm{Z}=20$ & $\mathrm{Z}=40$ & $\mathrm{Z}=56$ & $\mathrm{Z}=82$ & $\mathrm{Z}=90$ \\
\hline & $\underline{0 p-0 s}$ & $\underline{1 p-1 s}$ & $\underline{2 p-2 s}$ & $\underline{2 p-2 s}$ & $\underline{3 p-3 s}$ & $\underline{3 p-3 s}$ \\
\hline$<V_{P, T}>$ in $\mathrm{eV} \times 10^{-4}$ & 1.084 & 0.875 & 0.708 & 0.779 & 0.608 & 0.633 \\
\hline$i<V_{P}>$ in $\mathrm{eV}$ & 1.513 & 1.550 & 1.535 & 1.576 & 1.581 & 1.600 \\
\hline$\kappa^{(1)}$ & -8.2 & -6.4 & -5.3 & -5.6 & -4.4 & -4.5 \\
\hline & $\underline{0 p-1 s}$ & $\underline{1 p-2 s}$ & $2 p-3 s$ & $\underline{2 p-3 s}$ & $\underline{3 p-4 s}$ & $3 p-4 s$ \\
\hline$<V_{P, T}>$ in $\mathrm{eV} \times 10^{-4}$ & -0.400 & -0.378 & -0.388 & -0.465 & -0.376 & -0.409 \\
\hline$i<V_{P}>$ in $\mathrm{eV}$ & 1.294 & 1.435 & 1.441 & 1.485 & 1.508 & 1.527 \\
\hline$\kappa^{(1)}$ & 3.5 & 3.0 & 3.1 & 3.6 & 2.8 & 3.0 \\
\hline
\end{tabular}

